Revue d'Études Françaises

$\mathrm{N}^{\mathrm{o}} 24(2020)$

DOI : $10.37587 /$ ref.2020.1.04

\title{
AGNIESZKA WOCH
}

\section{(In)traduisibilité des variétés périphériques dans l'édition polonaise de Black Bazar d'Alain Mabanckou}

The Franco-Congolese novelist Alain Mabanckou made his debut with BleuBlanc-Rouge [Blue. White. Red], in 1998. In 2009, his seventh novel, Black Bazar, was published by Éditions du Seuil and one year later the Polish translation was published by Karakter publishing house. The novel, narrated by a sapeur and Parisian dandy, called by his entourage a Fessologue [Buttologist], is characterised by a succulent and incisive style and abounds in non-conventional expressions which render the translation very challenging. Our present contribution analyses the presence of slang in the Polish translation of Black Bazar and, examines afterwards the different translation techniques with a particular focus on the changes of register, compensation and undertranslation.

\section{Introduction}

Le roman Black Bazar d'Alain Mabanckou, qui constitue le corpus de notre étude, a été publié en 2009 aux Éditions du Seuil. Il s'agit du septième roman de l'auteur franco-congolais. La traduction polonaise de l'ouvrage est parue en 2010 aux Éditions Karakter.

Le roman, tout comme les autres ouvrages de Mabanckou, se caractérise par un style pittoresque, succulent et mordant et abonde en expressions non conventionnelles. Sa traduction dans une autre langue constitue donc un vrai défi pour le traducteur. Ce roman, finaliste du Grand Prix RTL-Livre, a pourtant déjà été traduit dans une quinzaine de langues et même adapté au théâtre au Lavoir Moderne Parisien, au Festival d'Avignon et au Los Angeles Theatre Center.

Black Bazar est l'histoire d'un " dandy africain de notre temps, amoureux des cols italiens et des chaussures Weston, qui découvre sa vocation d'écrivain au détour d'un chagrin d'amour. Naviguant entre complainte et dérision, il brosse avec truculence un tableau sans concession du monde qui l'entoure $»$ '.

\footnotetext{
${ }^{1}$ http://www.seuil.com/ouvrage/black-bazar-alain-mabanckou/9782020973373 (consulté 20.12.2019.)
} 
Dans cet article, nous nous proposons d'étudier la traduction des variétés périphériques dans l'édition polonaise de Black Bazar. Notre approche sera comparative : dans un premier temps, nous analyserons les termes non standard dans le texte source et dans le texte cible (la traduction en polonais) en nous penchant également sur les différentes techniques employées par le traducteur telles que le changement de registre et les jeux de compensation ; puis nous réfléchirons à l'(in)traduisibilité du corpus examiné.

\title{
1. La traduction des variétés périphériques dans Black Bazar
}

Nous partageons l'avis de Shango Lokoho qui attribue à la traduction « un rôle de médiation et de transfert culturel » en permettant «l'élargissement de l'horizon de réception des œuvres », tout comme la «facilitation des échanges culturels, des valeurs et des idées ». Shango Lokoho souligne l'importance de traduire "d'une littérature d'une langue à une autre» en donnant «une nouvelle vie à l'œuvre ». Il constate pourtant que le traducteur « se heurte non seulement à un univers nouveau, étranger, mais aussi à un langage ou à des langages particuliers, à des idiomes, à des idiomatismes, à des tics de langage, etc. » (Shango Lokoho, $2011: 86$ ). La traduction de la littérature francophone, selon Ezechiel Akrobou, est d'autant plus difficile qu'elle se situe «au carrefour entre l'écriture et l'oralité ». De plus,

\begin{abstract}
« traduire la littérature africaine francophone d'expression française [...] c'est tenir compte des paramètres contextuels socioculturels et linguistiques, issus de la production romanesque de l'auteur, c'est reconnaître en même temps que le parler maternel est irréductible à toute traduction. Traduire donc ce type de littérature revient à analyser l'articulation des pensées d'un message et leur reformulation dans une autre langue. Alors si le travail de traduction doit omettre la langue d'expression source, alors les innombrables références à la culture locale, le malinké, ne sauront prendre forme dans un projet de traduction » (Akrobou, $2012: 85)$.
\end{abstract}

En effet, la difficulté de traduire le roman Black Bazar réside dans la compréhension du contexte socioculturel dans lequel se trouve le protagoniste du roman, héritier de sa culture d'origine, mais également dans le choix du vocabulaire qui reflète la façon de parler des personnages et qui abonde en expressions imagées, caractéristiques d'ailleurs du style romanesque de Mabanckou, et en expressions relevant du langage non standard qui constituent l'objet de notre étude. 
Nous avons ainsi analysé les termes français non standard en les comparant à leurs équivalents polonais. Cela nous a permis d'élaborer une classification en quatre catégories avec leurs sous-catégories respectives, notamment :

- catégorie 1 a : le passage du français non standard (FNS) au polonais non standard (PNS);

- catégorie $1 \mathrm{~b}$ : le passage du FNS au PNS avec une atténuation de sens ;

- catégorie $1 \mathrm{c}$ : le passage du FNS au PNS avec un renforcement de sens ;

- catégorie 2 a : le passage du FNS au polonais standard (PS) ;

- catégorie $2 \mathrm{~b}$ : le passage du FNS au PS dicté par des déficits morphosyntaxiques ;

- catégorie 3 : le passage du français standard (FS) au PNS ;

- catégorie 4 : les autres cas (parmi lesquels la synonymie, les pertes de sens et les allusions).

\subsection{Le passage du français non standard (FNS) au polonais non standard (PNS)}

Le passage du FNS au PNS, classé dans la catégorie 1 a, peut être illustré par les exemples 1 à 17 . Notons que le traducteur a employé des équivalents non standard polonais assez fidèles au texte original :

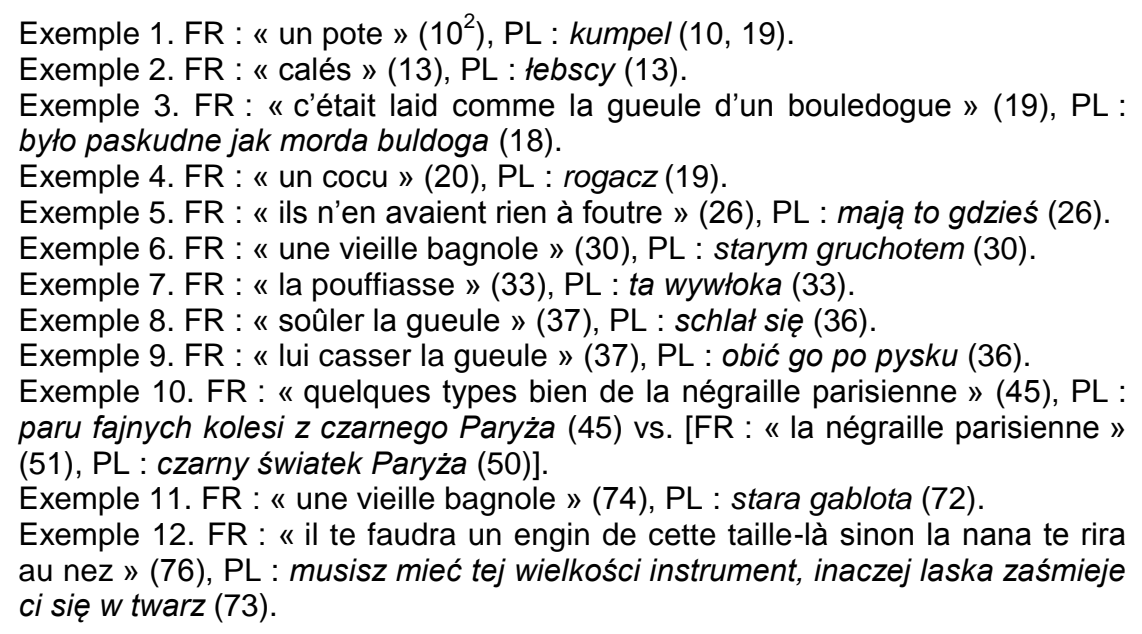

\footnotetext{
${ }^{2}$ Nous mettons entre parenthèses le numéro de page où nous avons relevé nos occurrences.
} 
Exemple 13. FR: «il se tapait les petites Congolaises » (79), PL : zaliczał dziewczynki z Konga (77).

Exemple 14. FR : « que je bande » (91), PL : kiedy mi staje (89).

Exemple 15. FR : «C'est de la merde là-bas ! » (97), PL : To syf ! (95).

Exemple 16. FR: "Arrête ton baratin, tu veux tirer ton coup, ça se voit!»

(171), PL : Przestań wciskać mi kit, chcesz sobie bzyknąć, to widać! (167).

Exemple 17. FR : "C'est ça, barre-toi, pauvre aliéné !»(245), PL : Jasne, spadaj, popaprańcu! (239).

Concentrons-nous sur la traduction de l'exemple 10 où "quelques types bien de la négraille parisienne » a été traduit en polonais littéralement par «quelques mecs de bien du Paris noir» et, plus loin, par «la petite société parisienne des noirs ». Cela montre que la volonté d'obtenir un texte plus varié et plus riche au niveau lexical peut parfois l'emporter sur le désir de rester au plus près du texte au niveau du registre.

Dans la catégorie $1 \mathrm{~b}$ (les exemples de 18 à 21), il existe une équivalence de sens entre les termes. Cependant, le texte cible est atténué en perdant ainsi ses connotations vulgaires.

Exemple 18. FR : "Qu'est-ce que vous avez foutu pendant ce temps ? ", PL : (15) A coście wyprawiali przez te wszystkie lata ? (15) [fabriquer].

Exemple 19. FR: "Ce vieux qui s'emmerde en brousse » (17), PL : Ten stary, który watkoni się w buszu (16) [flemmarder].

Exemple 20. FR: "Je ne rêvais que d'aller me taper les blondes, les rousses et les brunes. " (25), PL : Tylko marzę, by wyrwać jakąś blondynkę, rudą lub brunetkę. (25) [draguer].

Exemple 21. FR : « une putain de machine à écrire » (28), PL : w tę cholerna maszynę (28) [maudit].

Ainsi le verbe "foutre» dans l'exemple 18 est traduit par wyprawiać «fabriquer », s'emmerder par watkonić się «flemmarder» (ex. 19) tandis que « se taper» devient wyrwać « draguer» (ex. 19) et « une putain de machine», cholerna maszyna, « une machine maudite » (ex. 21).

En revanche, la catégorie $1 \mathrm{c}$ constitue l'inverse du phénomène précédent : les termes traduits en polonais sont considérablement plus marqués, avec un sens péjoratif. En témoignent les exemples 22 à 25 :

Exemple 22. FR : « espèce de Congolais » (41), PL : palant z Konga (40).

Exemple 23. FR : " espèce de Martiniquais » (41), PL : palancie z Martyniki (40).

Exemple 24. FR: " Je fermerais les yeux et je tirerais mon coup sans traces" (137), PL : Zamknąłbym oczy i zerżnałł bez żadnych konsekwencji (134). 
Exemple 25. FR : « Les Nègres t'emmerdent ! " (141), PL: Czarnuchy mają cię w dupie! (138).

Le verbe «se taper» (ex. 24) est traduit par le terme polonais zerżnąć qui est doté d'une très forte charge émotive et expressive, et d'un caractère fortement péjoratif et vulgaire. Or le «nègre », selon le Trésor de la langue française, semble être aujourd'hui " en voie de perdre ce caractère péjoratif, probablement en raison de la valorisation des cultures du monde noir $»^{3}$. Certes, dans notre cas, il apparaît dans un roman écrit par un écrivain franco-congolais. Pourtant, son équivalent polonais, czarnuch, reste un terme beaucoup plus marqué. Le dictionnaire de référence de la langue polonaise $P W N$ le qualifie de « méprisant » et « offensif » ${ }^{4}$.

\subsection{Le passage du français non standard (FNS) au polonais standard (PS)}

La deuxième catégorie, illustrée par des exemples 25-32, présente le passage du FNS au PS :

Exemple 25. FR : « casser les pieds » (27), PL : uwziąć się (27) [s'en prendre à qqn].

Exemple 26. FR : « toubibs » (34), PL : lekarze (33) [médecins].

Exemple 27. FR : «dare-dare » $(70,133), \mathrm{PL}$ : nie tracąc czasu (68) [sans perdre le temps].

Exemple 28. FR: "ce que je foutais dans ce bar » (86), PL : co właściwie porabiam $w$ tym barze (84) [faire].

Exemple 29. FR: "Mon ex s'est tirée au pays il y a quelques mois " (109), PL : Moja eks kilka miesięcy temu uciekła do kraju. (107) [s'enfuire].

Exemple 30. FR : "Ce monde est en train de foutre le camp " (119), PL : Ten świat ginie (117) [disparaître].

Exemple 31. FR : " Je m'en foutais de son passé » (145), PL : nie obchodzi mnie jej przeszłość (141) [Son passé ne me regarde pas].

Exemple 32. FR : « II était habillé comme un broussard avec sa cravate qui ressemblait à l'intestin grêle d'un pingouin. "(245), PL : Był ubrany tak, jakby wyszedt z buszu, a jego krawat przypominał jelito cienkie pingwina. (239) [comme s'il sortait de la brousse].

Les expressions non standard ont été traduites dans un équivalent polonais moins expressif, comme, par exemple, lekarze «médecins» au lieu de « toubibs » (ex. 26), nie tracac czasu « sans perdre de temps » au lieu de « daredare» (ex. 27), ou uciec «s'enfuir» qui a remplacé «se tirer» (ex. 29). Le

\footnotetext{
${ }^{3}$ https://www.cnrtl.fr/definition/nègre (consulté 20.01.2020.).

${ }^{4}$ https://sjp.pwn.pl/slowniki/czarnuch.html (consulté 20.01.2020.).
} 
terme « broussard», n'ayant pas d'équivalent en polonais non standard (ex. 32), a été traduit par une périphrase " comme s'il sortait de la brousse»; dans les autres cas, le traducteur aurait pu respecter le registre.

Contrairement au cas précédent, nous observons, dans la catégorie $2 \mathrm{~b}$, un changement de registre dû aux déficits morphosyntaxiques de la langue cible. Illustrons cette catégorie par les exemples 33 à 39 :

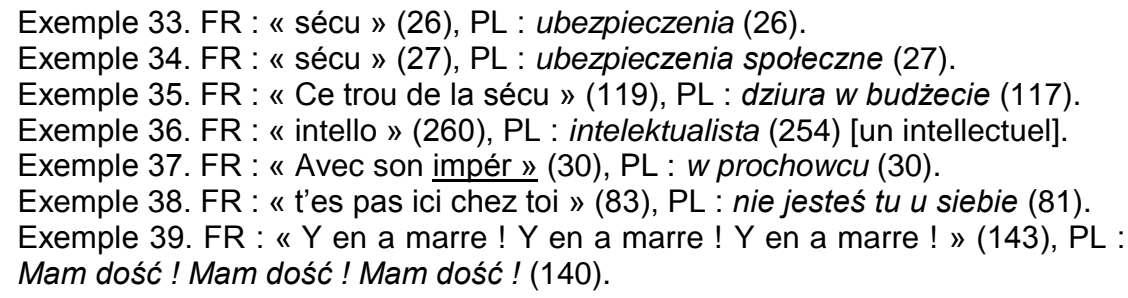

Dans ces cas, le passage du FNS au PS résulte des variations de structure et de grammaire entre les deux langues. En polonais, l'apocope est relativement rare dans les processus de création des termes non standard. De plus, dans la langue cible, on ne relève pas de procédés grammaticaux tels que l'élision interdite du pronom personnel, la suppression de «ne » dans la négation ou l'usage fréquent des ellipses qui inclut celles du sujet. En bref, le polonais n'utilise pas la syntaxe simplifiée et souvent approximative. Le terme « intello» (ex. 36) aurait peut-être pu être traduit par le diminutif ironique polonais inteligencik. En revanche, ube, apocope du mot ubezpieczenie (sécurité), serait resté un terme complètement opaque pour un lecteur polonais.

\subsection{Le passage du français standard (FS) au polonais non standard (PNS)}

La troisième catégorie est représentée par les exemples 40 à 47 :

Exemple 40. FR : " Laisse tomber tes histoires de t'asseoir et d'écrire tous les jours » (13), PL : Daj sobie spokój z tym siadaniem na tyłku i pisaniem co dzień (13) [s'asseoir sur son cul].

Exemple 41. FR : "Dans tes écrits » (20), PL: w twoich wypocinach (19) [élucubrations].

Exemple 42. FR: "Or tu vomis ta colère » (20), PL: a tylko rzygasz wściekłością (20) [dégueuler].

Exemple 43. FR : « Un petit voyou de rien » (135), PL : To zwykła szumowina (133) [racaille].

Exemple 44. FR : « (...) mais vraiment est-ce que c'est à cause d'une fille de ce genre que moi Macchabée j'irais en prison pendant deux ans, hein ?» 
(137), PL: (...) ale czy z powodu tego rodzaju dziewczyny ja, Truposz, poszedłbym na dwa lata do paki, hę ? (134) [taule].

Exemple 45. FR : "Ce T-shirt est à moi, ça coûte la peau des fesses et pas n'importe quelles fesses, même pas celles de Couleur d'origine » (142), PL: To mój T-shirt, jest wart więcej niż twoja dupa, a nawet dupa Pierwotnej Barwy (139) [cul].

Exemple 46. FR : "S'il me donnait un coup de tête » (142), PL : jeśli walnie mnie $z$ byka (139) [filer un coup de boule].

Exemple 47. FR : " *Fessologue » (13), Zadkolog (12) [pl. fam. zadek « petite croupe $»+$ suffixe -logue].

Elle regarde un procédé relativement peu fréquent pourtant présent dans le corpus analysé. Le passage du FS au PNS constitue possiblement une sorte de compensation de la part du traducteur quant au ton et au registre des termes présentés : ainsi le verbe «s'asseoir» (ex. 40) est renforcé dans le texte cible par le substantif tytek et devient "s'asseoir sur son cul », "vomir» devient rzygać « dégueuler» (ex. 42) et " prison» est traduit par le mot paka «taule» (ex. 44). L'exemple 47 concerne la traduction du sobriquet du héros principal de l'ouvrage, c'est-à-dire "Fessologue » qui a été traduit par Zadkolog. Le terme, composé du mot polonais zadek et du suffixe -log "-logue», a une connotation animale et désigne une " petite croupe ».

\subsection{Autres cas}

Dans la quatrième catégorie, nous avons regroupé les termes ou expressions tels que « gars » (ex. 48), « couillon» (ex. 49), « casser sa pipe» (ex. 50) ou le verbe «saper» (ex. 51) ainsi que le recours à une référence littéraire (ex. 52). Les exemples 48 à 50 témoignent du travail constant du traducteur pour employer des synonymes provenant de différents registres :

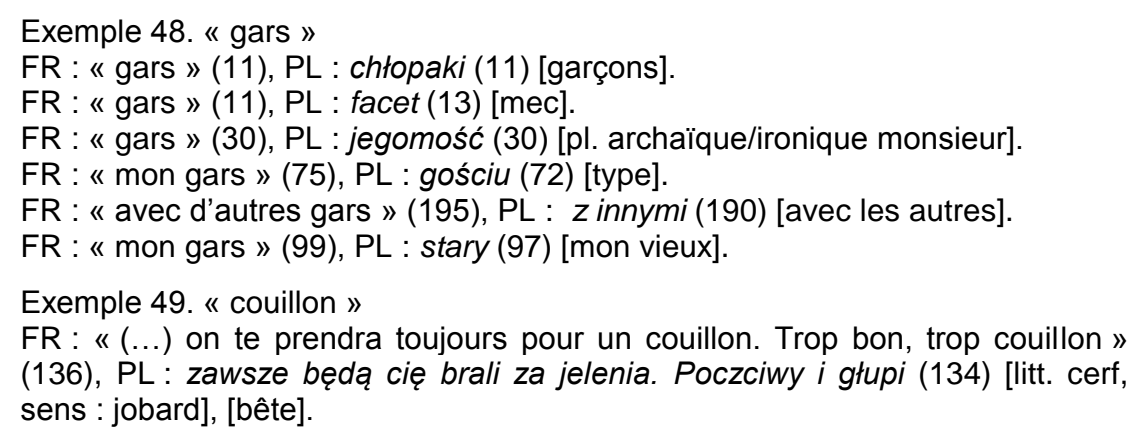




\section{Exemple 50. « casser la pipe "}

FR: "Je casserai ma pipe » (194), PL : kopnę w kalendarz (189) [pl. litt. donner un coup au calendrier « mourir »].

FR : «casser sa pipe » (254), PL : przekręcić się (248) [pl. litt. se tourner « mourir »].

FR : « casser sa pipe » (263), PL : wyzionąć ducha (257) [rendre l'âme].

Comment expliquer l'emploi systématique des synonymes dans la traduction polonaise, comme nous l'avons remarqué auparavant ? Peut-être pour rendre le texte cible plus varié au niveau lexical et éviter des répétitions qui ne sont pas absolument nécessaires, même si cela entraîne un changement de registre par rapport au texte de départ.

Dans le cas particulier du verbe « saper » (ex. 51), dans la langue cible, nous observons une perte en ce qui concerne le niveau de l'allusion culturelle à la mode vestimentaire des deux Congo. Le lecteur polonais non initié ne saisirait pas en effet l'allusion à «la société des ambianceurs et des personnes élégantes $»^{5}$. Le terme «saper» est donc traduit par un verbe familier picować się, « se parer », par l'expression mieć na sobie niezte ciuchy, " porter de belles fringues », ou bien par mieć wyczucie stylu, « avoir du style $»^{6}$.

Exemple 51. « saper»

FR: "Pour déposer les ordures dans un local est-ce qu'on est vraiment obligé d'être bien sapé comme si on se rendait à un mariage, hein ? » (28), PL : Naprawdę trzeba się picować jak do ślubu, żeby wyrzucić śmieci, hę ? (28) [se parer].

FR : " qui sont toujours bien sapés » (42), PL : którzy zawsze mają na sobie niezłe ciuchy (41) [porter de belles fringues].

FR : «Puisque mon goût pour la Sape était connu de tous, j'avais des clients en pagaille » (97), PL : Ponieważ moje wyczucie stylu było wszystkim znane, miałem mnóstwo klientów (95) [avoir du style].

Quant à l'exemple 52 :

Exemple 52. FR : «Elle ne va pas laisser congeler son pays-bas en plein hiver » (91), PL : Przecież nie zamrozi sobie polderu w samym środku zimy (89).

Pour l'expression à connotation sexuelle « laisser congeler son pays-bas », le traducteur a essayé de compenser la perte d'un jeu de mots («pays-bas» comme métaphore du sexe féminin) en le remplaçant par le mot polder

\footnotetext{
${ }^{5}$ https://fr.wikipedia.org/wiki/Soci\%C3\%A9t\%C3\%A9_des_ambianceurs_et_des_personnes_\%C 3\%A91\%C3\%A9gantes (consulté 08.09.2020.)

${ }^{6}$ Par contre, l'acronyme SAPE est traduit dans le texte par Stowarzyszenie Amantów, Pedantów $i$ Elegantów (42), « l'Association des Amants, des Pédants et des Élégants ».
} 
(étendue artificielle de terre gagnée sur l'eau, dont le niveau est inférieur à celui de la mer). Cependant, par l'emploi d'un terme géographique obscur pour un lecteur moyen, au lieu de compenser le jeu de mot, le traducteur a fait perdre le sens et le côté ludique de l'expression.

L'expression «le Roi des Cons» (ex. 53) est traduite dans la langue cible par Król Chujów ou chuj (pl. litt., vulg. « membre viril », fig. « salaud ») :

Exemple 53. FR : « le Roi des Cons » (210), PL : Król Chujów (204), chuj.

FR : " Hélas pour ce président, la chanson se murmurait sur toutes les lèvres, et on entendait dans la rue les gens siffloter, comme le chanteur de Sète, qu'il avait peu de chances qu'on détrône le Roi des cons, ce souverain pouvait donc dormir sur ses deux oreilles serein, tout le monde le suivrait docile, et qu'il était possible qu'on déloge le shah d'Iran mais qu'il y avait peu de chances qu'on détrône le Roi des cons... " (210), PL: Na nieszczęście dla prezydenta ta piosenka była na ustach wszystkich i na ulicy słychać było, jak ludzie pogwizdują niczym wassaty pieśniarz z Sète, że nie poradzisz nic bracie mój, kiedy na tronie siedzi chuj, i nic z oczu nie spędzi mu snu, wszyscy wciąż będą lizać dupę mu, że może z tronu spaść irański szach, lecz nie poradzisz nic, bracie mój, kiedy na tronie siedzi chuj... (204).

L'écart entre le texte original et la traduction s'explique par le fait que le traducteur reproduit presque fidèlement le texte de la chanson de Georges Brassens, «Le Roi », Król, en respectant la traduction déjà existante en polonais, datant des années 1980 et réalisée à l'époque par le groupe Zespót Reprezentacyjny formé par de jeunes étudiants. Le traducteur fait ainsi un clin d'œil en procédant aussi à une sur-traduction et en ajoutant l'adjectif wasaty, «moustachu» au «chanteur de Sète » original. Pourtant, une telle allusion ne sera comprise que par un lecteur cultivé et probablement plus âgé, l'exemple présentant la version polonaise de cette chanson n'étant pas connu du large public.

\section{Conclusions}

Dans le corpus analysé, nous avons donc observé le respect global du registre malgré le recours à l'atténuation ou au renforcement qui ne changent pas le caractère non standard de tous les passages cités. L'atténuation s'explique probablement par le fait que certains termes polonais, malgré leur correspondance lexicale avec les termes français, sont beaucoup plus marqués et, par conséquent, auraient été trop vulgaires. Quant au renforcement, il constitue peut-être une tentative de compenser la traduction en polonais des passages dans lesquels l'original non standard du texte n'a pas été rendu. 
Dans la grande majorité des cas, le transfert du non standard français au standard polonais est lié aux déficits lexicaux («broussard») et morphosyntaxiques de la langue cible (les cas d'apocope, d'ellipse, d'élision et de «ne » de négation). Ce dernier relève aussi d'une divergence fondamentale entre les deux langues : la syntaxe relâchée est généralement absente dans la structure polonaise.

Nous pouvons supposer que le phénomène contraire, c'est-à-dire le transfert du standard français au non standard polonais (d'ailleurs relativement peu fréquent dans le texte cible), vise à compenser, du moins partiellement, les pertes de la catégorie précédente.

Enfin, nous avons observé que les variétés périphériques dans le roman Black Bazar restent traduisibles malgré certaines pertes, non seulement au niveau du registre mais surtout au niveau culturel et ludique, qui auraient peutêtre pu être expliquées par une note en bas de page.

\title{
Bibliographie
}

AKROBOU Ezechiel (2012), "Traduire la littérature africaine francophone, entre oralité et écriture : le cas du roman Les soleils des indépendances, d'Ahmadou Kourouma », Estudios de Traducción, vol. 2, p. 77-86. DOI : 10.5209/rev_ESTR.2012.v2.38979

MABANCKOU Alain (2009), Black Bazar, Paris, Éditions du Seuil.

MABANCKOU Alain (2010), Black Bazar, Kraków, Karakter.

MESCHONNIC Henri (1999), Poétique du traduire, Paris, Verdier.

SHANGO LOKOHO Tumba (2011), «Du multilinguisme et de la traduction des littératures africaines ", in : Questions de méthode in Traduction \& Littérature multilingue (K. Alfons Knauth éd.), Hamburg, Lit Verlag, p. 68-94.

www.seuil.com/ouvrage/black-bazar-alain-mabanckou/9782020973373

(consulté 01.12.2019.).

www.cnrtl.fr (consulté 20.12.2019.).

www.sjp.pwn.pl/sjp/online (consulté 22.12.2019.).

\author{
AGNIESZKA WOCH \\ Université de Łódź \\ Courriel : agnieszka.woch@uni.lodz.pl
}

